\title{
Vinculación de la economía social y el crecimiento verde en Costa Rica bajo un estudio de casos múltiples
}

\author{
Linking the social economy and green growth in Costa Rica \\ under a multilpe case study
}

DOI: $10.22458 /$ rna.v $12 \mathrm{i} 2.3813$

\author{
Dr. Manuel Campos-Rudin \\ Universidad Estatal a Distancia, Estudiante doctoral, \\ Sistema de Estudios de Posgrado, San José, Costa Rica; \\ mcamposrudin@gmail.com, https://orcid.org/o000-0002-0350-8569
}

Fecha de recepción: 15 de julio de 2021

fecha de aceptación: 12 de agosto de 2021

\section{RESUMEN}

Costa Rica es líder en temas ambientales y posee una economía social consolidada. La economía social y el crecimiento verde pueden ser una combinación viable para lograr la sostenibilidad. Esta investigación busca conocer, por medio de un estudio de casos múltiples con cuatro cooperativas, una asociación solidarista y una caja de ahorro, si las acciones verdes emprendidas y los beneficios sostenibles obtenidos han generado impactos positivos en estas organizaciones; indaga si estos procesos fueron financiados mediante herramientas financieras verdes. Los resultados obtenidos demostraron que sí hay una vinculación del crecimiento verde con los casos estudiados, aunque se detectó que hay aspectos por mejorar, como la protección de la biodiversidad. Además, se perciben beneficios por las acciones verdes realizadas, pero se observa que hay un limitado uso de herramientas financieras verdes, lo cual implica que se podría potenciar el crecimiento verde si se implementaran.

\section{ABSTRACT}

Costa Rica is a leader in environmental topics and has a consolidated social economy. The social economy and green growth can be a viable combination to reach sustainability. This research seeks to know, through a study of multiple cases with four cooperatives, one solidarity association and a savings bank, if the undertaken green actions and the sustainable benefits that were obtained have promoted positive impacts on these organizations; inquires if these processes were financed through green financial tools. The obtained results showed that there is a link between green growth and the cases studied, although it was detected that there are aspects to be improved, such as the protection of biodiversity. In addition, benefits are perceived for the green actions carried out, but it is observed that there is a limited use of green financial tools, which implies that green growth could be enhanced if they were implemented.

\section{RESUME}

Le Costa Rica est leader en matière environnementale et possède une économie sociale consolidée. L'économie sociale et la croissance verte constituent une combinaison viable pour parvenir à la durabilité. Cette recherche vise à connaître, au moyen d'une étude de cas multiples avec quatre coopératives, une association solidariste et une caisse d'épargne, si les actions vertes mises en place et les bénéfices durables obtenus ont produit des impacts positifs sur ces organisations ; demande si ces processus ont été financés à travers d'outils financiers verts. Les résultats obtenus montrent qu'il existe un lien de croissance verte et les cas étudiés, même si on remarque qu'il y a des domaines à améliorer comme la protection de la biodiversité. En outre, des avantages sont perçus grâce aux actions vertes réalisées, mais on constate que l'utilisation des outils financiers verts est limitée, ce qui signifie que la croissance verte pourrait être renforcée s'ils étaient mis en œuvre.

\section{RESUMO}

A Costa Rica é líder em questões ambientais e possui uma economia social consolidada. A economia social e o crescimento verde podem ser uma combinação viável para atingir a sustentabilidade. Esta investigação busca conhecer, por meio de um estudo de casos múltiplos com quatro cooperativas, uma associação solidária e uma caixa de poupança, se as ações verdes empreendidas e os benefícios sustentáveis obtidos geraram impactos positivos nessas organizações; indaga se esses processos foram financiados por meio de ferramentas financeiras verdes. Os resultados obtidos demostraram que existe uma ligação entre o crescimento verde e os casos estudados, embora tenha sido detectado que existem aspectos a melhorar, como a proteção da biodiversidade. Além disso, são percebidos benefícios pelas ações verdes realizadas, mas observase que há um uso limitado de ferramentas financeiras verdes, demostrando que o crescimento verde poderia ser potencializado se fossem implementadas.

\section{MOTS CLES :}

DURABILITE, COOPERATIVES,

SOLIDARISME, BANQUE

ETIQUE, CHANGEMENT

CLIMATIQUE, RESSOURCES

ECOSYSTEMIQUES, FONDS

FINANCIERS VERTS
PALAVRAS-CHAVE: SUSTENTABILIDADE, COOPERATIVAS SOLIDARISMO, BANCO ÉTICO, MUDANÇAS CLIMÁTICAS, RECURSOS DO ECOSSISTEMA, FUNDOS FINANCEIROS VERDES 


\section{INTRODUCCIÓN}

Los modelos de asociación vinculados con la economía social (ES) están unidos a la economía costarricense desde mediados del siglo XX. En este contexto se observa la aparición de la primera cooperativa en Costa Rica llamada CoopeVictoria R.L., el 12 de octubre de 1943 (CoopeVictoria, 2019). También, Costa Rica, de la mano de Alberto Martén, en 1947 introdujo la propuesta vinculada a la ES llamada solidarismo, donde trabajadores y patrones constituían una organización con los aportes de ambos grupos para lograr una mejor estabilidad financiera de los asociados (CONASOL, 2021). Esto, en el modelo de cajas de ahorro, ocurrió con la creación de la Junta de Ahorro y Préstamo de la Universidad de Costa Rica (JUNTA UCR) en 1940. Consistía en una caja de ahorro donde los trabajadores realizaban un aporte obligatorio y este era compensado con un aporte patronal, mientras hubiese relación laboral (Contreras, 2012). Se puede deducir que la ES tuvo un proceso dinámico en la década de los años cuarenta, vinculada con las reformas sociales de la época en Costa Rica. Todo este proceso potenció la generación de nuevas organizaciones vinculadas a la ES (Quesada, 2008).

La ES ha impulsado el crecimiento económico de Costa Rica y aborda sectores como el agrícola, servicios financieros, energéticos y otros, los cuales permiten dar dinamismo en las regiones del país donde esta se ha logrado consolidar. Los retos que dichas organizaciones afrontan en el nuevo milenio involucran los efectos del cambio climático (CC) y cómo realizar acciones que potencien un crecimiento sostenible. En la actualidad, Costa Rica ha planteado diversas acciones para afrontar el CC como, por ejemplo, reducir las emisiones de gases de efecto invernadero, protección de la biodiversidad, inducir la bioeconomía y potenciar el crecimiento verde desde finales del siglo XX y durante este nuevo milenio. Algunas de las acciones concretadas son:

b) Programa de pagos por servicios ambientales gestado por el Fondo Nacional de Financiamiento Forestal (Fonafifo, 2021).

c) Declaración para la carbono neutralidad (Dobles, 2021).

d) Programa para descarbonización (Minae, 2018).

e) Programa para impulsar la bioeconomía (Micitt, 2020).

El caso de los pagos por servicios ambientales en Costa Rica tiene más de 20 años de existencia. Estos han sido tomados como un caso de éxito internacional por su impacto en la recuperación y protección de áreas de bosque y estímulos para la reforestación, donde han obtenido fondos del Banco Mundial y la fijación de impuestos a los combustibles (Sánchez y Navarrete, 2017). Con respecto a la carbono neutralidad declarada en la administración del presidente Oscar Arias en 2006, los resultados y propuestas están sujetos a debate en los alcances que ha tenido (Baltodano, 2008), pero no deja de ser un esfuerzo país necesario. Los otros dos programas mencionados, el de descarbonización y el de bioeconomía, fueron propuestos por el presidente Carlos Alvarado entre 2018 y 2020, ambos de gran visión, pero sin contenidos financieros claros para que puedan ser ejecutados y sin una posible vinculación con los ya existentes en el país.

El presente trabajo realizó un estudio de casos múltiples con seis organizaciones de la ES de Costa Rica, con el objetivo de conocer los tipos de acciones concretas en favor del crecimiento verde, los beneficios obtenidos por estas acciones verdes en pro de la sostenibilidad e indagar la forma en que financiaron estos procesos. Todo esto con el fin de analizar y comprender cómo el sector de ES afronta los retos del CC y la sostenibilidad ambiental, económica y social dentro del entorno del crecimiento verde.

\section{FUNDAMENTACIÓN TEÓRICA}

Lograr la sostenibilidad ante el CC y el reto que implica el manejo racional de los ecosistemas debe ser un tema presente en las organizaciones, sean públicas, fundaciones, de capital privado con fines lucrativos (nacionales o transnacionales), vinculadas a la ES o de cualquier otro tipo. Una forma en que las organizaciones han tratado de lograr un proceso sostenible es bajo la gestión ambiental y su accionar es definido por Massolo (2015) como:

El conjunto de acciones y estrategias mediante las cuales se organizan las actividades antrópicas que influyen sobre el ambiente con el fin de lograr una adecuada calidad de vida previniendo o mitigando los problemas ambientales. Partiendo del concepto de desarrollo sostenible, se trata de conseguir el equilibrio adecuado para el desarrollo económico, crecimiento de la población, uso racional de los recursos y protección y conservación del medio ambiente. (p. 11) 
Las emisiones de gases de efecto invernadero, como fuentes antropocéntricas generadoras del CC, no dejan de crecer (Peters et al., 2019) y las economías tienden a acoplar su crecimiento - medido por el producto interno bruto (PIB) - a las emisiones de carbono y sus equivalentes (OCDE, 2017); Costa Rica no es la excepción (Campos-Rudin, 2019). Debido a estos resultados, se requieren acciones diferentes para descarbonizar las economías y buscar la sostenibilidad. Una estrategia es el crecimiento verde $(\mathrm{CV})$ y las organizaciones de la ES pueden ser un pilar para potenciar este proceso.

\section{El crecimiento verde y la economía social}

El CV tiene varias definiciones y orientaciones. Para el presente estudio se tomó la definición que brinda la Organización para la Cooperación y el Desarrollo Económico (OCDE), organización a la cual Costa Rica fue admitida en 2021. La OCDE (2011) define que el:

Crecimiento verde significa fomentar el crecimiento y el desarrollo económicos y al mismo tiempo asegurar que los bienes naturales continúen proporcionando los recursos y los servicios ambientales de los cuales depende nuestro bienestar. Para lograrlo, debe catalizar el desarrollo sostenible, inversión e innovación que apuntalen el crecimiento sostenido y abran paso a nuevas oportunidades económicas. (p. 17)

El CV se vincula con los denominados Objetivos de Desarrollo Sostenible del siglo XXI (ODS), entre los cuales se pueden citar algunos como el objetivo 12, que menciona la producción y consumo responsables, el 13 aborda la acción por el clima, el 14 la vida submarina, el 15 la vida de ecosistemas terrestres, mientras que el 16 y 17 se vinculan con el desarrollo sostenible (Naciones Unidas, 2015). Estos objetivos influyen sobre el CV al establecer un uso sostenible de los recursos ecosistémicos para esta y las futuras generaciones.

La ES se puede integrar de forma activa con el CV y los ODS del siglo XXI. La comisión científica del Centro Internacional de Investigación e Información sobre la Economía Pública, Social y Cooperativa (CIRIEC-España), citada por Montolio (2002), define la ES como un:

Conjunto de empresas privadas que actúan en el mercado con la finalidad de producir bienes y servicios, asegurar o financiar y en las que la distribución del beneficio y la toma de decisiones no están ligadas directamente con el capital aportado por cada socio, correspondiendo un voto a cada uno de ellos. (p. 19)

La CIRIEC-España añade que esto vincula a aquellas organizaciones de productores, no de mercados privados y no controladas por la administración pública, donde se dirigen acciones específicas para un grupo de consumidores o personas (Montolio, 2002). La ES posee, según Salinas (2003), una serie de elementos que la definen y complementan:

i. La Economía Social se definirá como aquella actividad económica que tenga por objeto trabajar para sus miembros y usuarios y para la sociedad... ii. Las personas están por encima del capital... iii. La solidaridad es el motor de la Economía Social... iv. Es una concepción de empresa en sentido amplio, referido a la gestión de recursos disponibles para la eficaz consecución de los objetivos sociales. v. Subyace en todo ello un eje económico y colectivo que tiende a concebir este tipo de actividad en valores de riqueza colectiva (no de enriquecimiento individuales). (p. 99)

A partir de la cita, la ES se puede vincular a varios de los ODS y, de manera consecuente, no puede lograr un riqueza colectiva sostenible en este nuevo milenio si no se gestiona un CV junto con otras tendencias de gestión, como la bioeconomía circular, que pueden ser acopladas integralmente a la ES (García-Barquero et al., 2018).

En Costa Rica la ES tiene organizaciones activas desde mediados del siglo XX, las cuales son posibles de constatar por su rápido crecimiento en cantidad (Quesada, 2008). El solidarismo brinda, a las empresas de capital lucrativo y a sus colaboradores, un modelo gestado en la economía costarricense, donde existen consideraciones a favor y en contra de este (Soto, 1987). De la Ley de Asociaciones Solidaristas N. ${ }^{\circ} 6970$ se extraen puntos relevantes que marcan su accionar. El solidarismo y los fondos que se inyectan a la asociación sirven para aliviar necesidades financieras o sociales de los afiliados, los cuales la conforman únicamente los colaboradores de la empresa. Las herramientas financieras que brindan poseen tasas favorables bajo principios éticos. El dinero aportado mínimo por cada colaborador se acuerda entre los afiliados y el equivalente depositado por la empresa, el dinero solo puede ser 
retirado en su totalidad cuando se termina la relación laboral con la empresa madre. Este tipo de modelo, además de apoyar a los colaboradores de la empresa en temas económicos y sociales, busca eliminar o alivianar posibles conflictos obrero-patronales (ASOUNA, 2021).

DentrodelaESseubicalabancaética.Labancaéticabuscadiferenciarsedelabancatradicionalquesolopersigueresultados técnicosy, deforma tradicional,procedeadardinerobajohipotecas quecubranel 100\%delcréditoy anegocios tradicionales con resultados a corto plazo. De modo que los ahorrantes no pueden definir dónde realizar inversiones. Según Ruiz y Retolaza (2007), banca ética se define: "por los sectores o tipo de actividades donde en las que colocan dinero (activo) y por la opción de crear conciencia y compromiso entre los depositarios, permitiéndoles decidir el destino de sus fondos (pasivos)" (p. 1104).

La banca ética no se enfoca en brindar únicamente microcréditos para empresas sociales. Se vincula con organizaciones que buscan potenciar la filantropía, procesos mediante los cuales las corporaciones, bajo la responsabilidad social corporativa, ayudan a grupos en riesgo, con el fin de que estos puedan desarrollar algún tipo de emprendimiento. Aunque las iniciativas microfinancieras dieron a Yunus el Premio Nobel de la Paz en 2006 por la creación del Grameen Bank, el querer emular el modelo del Grameen Bank en otro contex to no ha dado los resultados esperados (Sasia, 2008). En Costa Rica un modelo de banca ética es el Instituto Nacional de Vivienda y Urbanismo (INVU), una institución que brinda bonos de vivienda, planes de ahorro y crédito con tasas preferenciales menores a la banca tradicional (INVU, 2021). Otra forma de visualizar la banca ética son las cajas de ahorro y préstamo, las cuales son usadas en la economía social para ayudar a los afiliados en temas financieros y sociales, como el caso de la JUNTA UCR (2021).

Las cooperativas son el tercer modelo asociativo analizado en esta investigación. Según Martínez (2015), "la cooperativa es un origen, un recurso para obtener de forma compartida la satisfacción de una necesidad común. Es una alternativa a las posibilidades o la falta de posibilidades, y una aplicación más de la obtención de la fuerza mediante la unión” (p. 35). De forma más detallada está la definición de cooperativismo planteada por la Alianza Cooperativa Internacional (ACI, 2021), como "una asociación de personas que se han unido de forma voluntaria para satisfacer las necesidades y aspiraciones económicas, sociales y culturales en común mediante una empresa de propiedad conjunta y de gestión democrática”.

Las cooperativas tienen siete principios que las rigen:
1) Afiliación voluntaria y abierta
2) Control democrático de los miembros
3) Participación económica de los socios
4) Autonomía e independencia
5) Educación, formación e información
6) Cooperación entre cooperativas
7) Sentimiento de comunidad (ACI, 2021).

En el séptimo principio es donde se puede vincular el CV a las cooperativas, pues en este se busca el desarrollo sostenible de sus comunidades, por medio de las políticas y gestiones avaladas por sus afiliados o miembros. El ambiente cambiante y con retos para lograr la competitividad sostenible obligan a las cooperativas a buscar procesos de innovación (Marcuella y Saz, 2008). Además, las lleva a implementar en forma continua modelos de responsabilidad social y acciones ambientales para lograr resistir en momentos de crisis. Estas cooperativas han logrado mostrar procesos de resiliencia mayores que otras organizaciones (Martínez, 2015). Estos procesos de innovación y acciones para lograr el CV deben impulsarse por medio de herramientas financieras verdes (HFV). 


\section{Herramientas financieras verdes para impulsar el crecimiento verde}

La ES busca una equidad para que los beneficios económicos se distribuyan de forma homogénea en la sociedad, se logra así un balance entre lo económico y social, esto ante la creciente inequidad del aumento económico en las naciones (Ben-Ner, 2018), pero para lograr un proceso sostenible se ocupa control sobre la parte ambiental. ${ }^{1}$ La gestión o desarrollo de acciones concretas para lograr afrontar el reto de la sostenibilidad ambiental al buscar un CV $\mathrm{y}$ afrontar el CC requiere de insumos financieros.

Nicolas Stern, en su conocido Informe Stern, sobre la economía y el CC publicado en el año 2006, realizado por solicitud del gobierno de Inglaterra, detalla que los costos económicos y sociales del CC se estiman por ton de $\mathrm{CO}_{q}$ en USD $\$ 85$. Este informe indica que para reducir o contener en $1^{\circ} \mathrm{C}$ el calentamiento global y para estabilizar las emisiones entre 500-550ppm de $\mathrm{CO}_{2}$, esto equivaldría para el año 2050 al $1 \%(-2 \%,+5 \%$, rangos) del PIB si se tomaran las medidas oportunas a tiempo (Stern, 2010).

Otra forma de visualizar el impacto del CC es por medio de los cisnes verdes. Bolton et al. (2020) publica su informe The Green Swan, donde manifiesta el arribo de una crisis ambiental que tendrá efectos ambientales, sociales y económicos de dimensiones globales mayores a los conocidos, en la cual el CC es uno de los promotores. Los autores señalan que a diferencia de las crisis financieras generadas por catástrofes naturales (por ejemplo, terremotos), actos de terrorismo o especulación, en las que hay fuertes afectaciones económicas o sociales temporales, denominados cisnes negros; los cisnes verdes pueden poner en riesgo la misma existencia de la humanidad y sus efectos generarían fuertes reacciones en cadena, complejas e impredecibles para el ambiente, la economía y la sociedad. La actual crisis de salud y económica generada por el covid-19 podría ser considerada un cisne verde.

La sostenibilidad ambiental para ser atendida y evitar los cisnes verdes, requiere de protección de la biodiversidad, protección y uso racional de los ecosistemas, reducción de gases de efecto invernadero, planes de mitigación y adaptación ante el CC, ecoeficiencia, planes de gestión ambiental, educación ambiental, inversión en energías limpias, protección del recurso hídrico, por citar las más relevantes (The Board of the Millenium Ecosyste Assesment, 2005). No está únicamente vinculado a controlar las emisiones de gases de efecto invernadero y contrarrestar el CC.

La generación de diversas HFV es requerida para implementar un proceso de CV. En los informes de Finanzas para la Biodiversidad (BIOFIN) se pueden localizar datos sobre la gran diversidad de herramientas financieras que pueden implementarse para apoyar un CV (PNUD, 2018).

BIOFIN realizó un exhaustivo análisis de instrumentos financieros disponibles, con el objetivo de aplicar medidas vinculadas con la preservación de la biodiversidad y la sostenibilidad de los recursos ecosistémicos. De manera global, este programa detecto más de 150 soluciones financieras (PNUD, 2018). Los instrumentos financieros fueron separados por BIOFIN en las siguientes categorías:

1) Préstamos tradicionales y concesionales. Bajo ese esquema se buscaba capital de trabajo para que los bancos ofrecieran créditos para realizar procesos verdes. El Banco Interamericano de Desarrollo ha dado préstamos concesionales a Latinoamérica para enfrentar el CC.

2) Subsidios bilaterales y multilaterales. Estos son fondos económicos brindados por organismos internacionales para mitigar o adaptarnos al CC.

3) Canjes de deuda. Estos mecanismos, en la parte ambiental, una nación con deudas puede canjear su deuda con el acreedor al gestionar proyectos ecológicos o de conservación.

4) De gestión de riesgo. Es cualquier instrumento que implique la transferencia de riesgos entre dos o más partes. Puede ser el pago de un seguro o un acuerdo específico entre las partes.

5) Fiscal. Se refiere a la generación de ingresos para lograr un cometido, basado en reformas tributarias como la generación de un impuesto a la utilización de plásticos de un solo uso. Tiene la desventaja de que, en naciones en vías de desarrollo, pueden ser un elemento que promueva la evasión fiscal, o simplemente su efecto, en general, afectaría a las clases más pobres.

1 Para esta investigación el concepto ambiental se refiere a los ecosistemas, la biodiversidad y los aspectos biológicos. No se contempla como ambiental las relaciones obrero-patronales o incidentes laborales dentro de las organizaciones. 
6) De mercado. Cualquier instrumento que implique transacciones o precios de mercado que influya directamente sobre estos, se dan las condiciones de oferta y demanda de un producto o servicio. Por ejemplo, Nestlé en Francia paga a los agricultores por la conservación del agua. Los mercados de carbono pueden ser incluidos en este rubro.

7) Bonos verdes. Representan menos del $1 \%$ del mercado global de bonos, pero se hallan en crecimiento (Clapp, 2018). Pueden ser incluidos dentro de los instrumentos de mercado, pero se separan por su gran versatilidad si logran en un futuro tener una amplia participación en el mercado bursátil.

8) Normativo. Implica la generación de multas o castigos financieros a las personas y organizaciones que generen daños al ambiente y la biodiversidad. Este incluye la generación de leyes y reglamentos para su correcta ejecución.

Las HFV en Costa Rica se pueden ubicar en la banca estatal y la banca privada, muchas de estas entidades ofrecen créditos diferenciados para optar por la compra de productos vinculados con la sostenibilidad ambiental. No es de interés de esta investigación contrastar las tasas de interés y condiciones de los créditos con los créditos hipotecarios o líneas de crédito convencionales, por lo cual no se discutirá si estos créditos verdes son o no competitivos para las organizaciones. Por este motivo solo se presenta en la tabla 1 un extracto de organizaciones bancarias y no bancarias que han canalizado HFV en Costa Rica.

\section{Tabla 1. Entidades bancarias, cooperativas, cámara de industrias y otras entidades financieras}

\begin{tabular}{|l|l|}
\hline \multicolumn{1}{|c|}{ Entidad financiera / Organización de la ES } & Nombre de la HFV \\
\hline Bolsa Nacional de Valores (BNV) & BNV sostenible \\
Banco Nacional de Costa Rica & BN pyme verde \\
Banco de Costa Rica & Eco-créditos \\
Banco Popular & Eco-créditos \\
Banco Promerica & Créditos Verdes \\
Bac San José & Green Pymes \\
Banco Scotiabank & No tiene una línea específica \\
Banco Lafise & Eco-créditos \\
Fundecooperación & Apoyo general a proyectos verdes \\
Cámara de Industrias de Costa Rica (CICR) & Apoyo amplio a proyectos verdes* \\
JUNTA UCR & Tecnológico \\
& \\
\hline
\end{tabular}

* La CICR no es una entidad financiera, es una entidad que busca gestar alianzas con bancos extranjeros y nacionales.

Fuente: Información desplegada en las páginas web oficiales de las diferentes entidades mencionadas.

Con respecto a la venta o comercialización de bonos carbono y bonos de sostenibilidad, en Costa Rica existe la opción de financiar proyectos con un fuerte componente verde, por medio de la BNV (2021). Además, existen fondos semilla para emprendedores y pequeñas empresas para realizar mejoras en temas de sostenibilidad, con lo cual en el país se disponen de HFV para apoyar el desarrollo de proyectos verdes (Procomer, 2021).

\section{METODOLOGÍA}

\section{Tipo de estudio y muestra de estudio}

Esta investigación se realiza bajo el paradigma cualitativo, como un estudio de casos múltiples explicativo. La selección de la muestra no probabilística se gestó bajo el criterio experto de investigador; este tipo de muestreo no probabilístico requiere que el investigador tenga conocimiento para elegir los casos de forma asertiva (MartínezSalgado, 2012). Para la elección de los casos de estudio, el investigador planteó como base dos criterios: ser organizaciones con más de 30 años de operar en Costa Rica y haber demostrado acciones concretas en el tema ambiental, en la preservación de los ecosistemas, energías alternativas o haber obtenido certificaciones verdes. 
Luego, se procedió a invitar a las organizaciones para participar, aquellas que respondieron fueron preseleccionadas. Posterior a esto, el investigador visitó el ecosistema en que estaban inmersas las organizaciones para validar si estas tenían un impacto en sus afiliados y si los productos o servicios tenían componentes verdes en el sitio, además de revisar en sus páginas web su historia y acciones por la sostenibilidad social, ambiental y económica. Las organizaciones seleccionadas dentro del estudio fueron cuatro cooperativas (CoopeTarrazú R.L., Coopedota R.L., Coopesantos R.L., y CoopeVictoria R.L.), una asociación solidarista ASOELAGAR y la JUNTA UCR. Se trató de establecer contacto con cooperativas vinculadas al sector financiero, pero no se logró obtener respuestas favorables de interés para participar en el estudio de casos.

\section{Recolección de datos}

Se realizaron encuestas a directivos y ejecutivos de las organizaciones seleccionadas bajo el formato de escala Likert. Por medio de 49 variables cerradas se documentó el impacto de las acciones y beneficios derivados de estrategias vinculadas al CV, las cuales fueron agrupadas en tres ejes temáticos: acciones concretas para el CV, herramientas financieras (HF) y los beneficios percibidos por la organización en lo social, económico y ecológico o ambiental. Todos los ejes se subdividieron en varios conceptos para el análisis. El tema ambiental, para esta investigación, se vinculó con los ecosistemas, recursos ecosistémicos y biodiversidad.

Las entrevistas fueron realizadas entre febrero de 2020 y febrero de 2021 . Aquellas realizadas antes de la pandemia por el covid-19 o en momentos de muy baja incidencia, se hicieron de forma presencial, otras fueron ejecutadas de manera virtual, mediante la plataforma Zoom.

\section{Variables de estudio según los ejes de investigación}

El eje acciones concretas en CV se subdivide en tres conceptos: el ecológico, el de energía y el de certificaciones verdes (en la tabla 2 se listan las variables utilizadas). El eje HF se divide en dos conceptos: las herramientas financieras convencionales (HFC) y las HFV. Las HFC se consideran todos los mecanismos financieros regulares basados en hipotecas, créditos comerciales y cualquier otro no diferenciado del tema verde. Las HFV son mecanismos vinculados con bonos de carbono, pagos por servicios ambientales, créditos específicos para temas verdes y fondos específicos para acciones verdes. El detalle de las variables se observa en la tabla 2.

Eje sostenibilidad, beneficios económicos, sociales y ambientales derivados del CV se basa en tres conceptos: el ambiental, el económico y el social. En este eje temático se buscó estimar los impactos que las acciones verdes y el uso de HFV han tenido sobre la sostenibilidad de la organización; incluso se contempló una variable sobre el covid-19.

\section{Análisis de datos obtenidos}

Todas las variables se analizaron bajo la escala de Likert, donde la valoración del impacto de las medidas adoptadas se codificó de la siguiente forma: 0 , no aplica; 1, no tuvo; 2, bajo; 3, regular; 4, mediano, y 5, alto. La información colectada en las organizaciones se ponderó y se procedió a graficar los datos para cada una de las variables agrupadas por eje temático y por concepto, con la finalidad de obtener los resultados individuales para su análisis.

Para efectuar un análisis global y generar un posible indicador para todas las organizaciones, se realizó el diseño y cálculo de tres tipos de índices de impacto basados en las respuestas obtenidas en la escala de Likert, al cuantificar porcentualmente el impacto de cada variable. Para cada variable analizada el puntaje máximo era 5, el cual es equivalente a alto impacto, de esta forma si las seis organizaciones analizadas respondieron como alto impacto se obtiene el puntaje máximo de 30 puntos (5 pts. x 6 respuestas). Este razonamiento se aplicó para generar un índice de impacto por cada variable y se contabilizó el valor porcentual. 


\section{Tabla 2. Variables analizadas en las entrevistas para los casos de estudio, agrupadas por eje temático}

\begin{tabular}{|c|c|c|}
\hline Eje acciones CV (n=16) & Eje HF $(n=18)$ & $\begin{array}{c}\text { Eje sostenibilidad, beneficios } \\
\text { ambientales, económicos y } \\
\text { sociales }(n=15)\end{array}$ \\
\hline Concepto: Ecológico & Concepto: HFC (no verdes) & Concepto: Ambiental \\
\hline $\begin{array}{l}1 \text { Apoyo a socios o afiliados en temas } \\
\text { verdes. }\end{array}$ & 1 Beneficio fiscal no enfocado a CV. & 1 Reducción de contaminación ambiental. \\
\hline $\begin{array}{l}2 \text { Construcción de infraestructura } \\
\text { ecoeficiente. }\end{array}$ & 2 Capital de los asociados. & $\begin{array}{l}2 \text { Reducción riesgos ambientales en la } \\
\text { organización. }\end{array}$ \\
\hline 3 Empaques ecológicos. & 3 Capital propio de la organización. & 3 Se logró proteger la biodiversidad. \\
\hline 4 Manejo de residuos. & 4 Préstamos convencionales de la banca. & \\
\hline 5 Manejo del recurso hídrico. & 5 SBD en forma general. & Concepto: Económico \\
\hline 6 Para protección de la biodiversidad. & 6 Transferencias de Infocoop generales. & 4 Crecimiento de mercado generado por CV. \\
\hline 7 Producción más limpia. & Concepto: HFV & 5 Impacto de CV en el flujo de caja. \\
\hline 8 Producción verde o sostenible. & 7 Bonos de carbono. & 6 Impacto de CV en los ingresos netos. \\
\hline & 8 Créditos bancarios para ambiente. & 7 Mejor productividad. \\
\hline Concepto: Energía & 9 Créditos bancarios para energías alternativas. & 8 Nuevos mercados producto del CV. \\
\hline 9 Biocombustibles. & $\begin{array}{l}10 \text { Créditos internacionales para energías } \\
\text { alternativas. }\end{array}$ & \\
\hline 10 Calentadores solares. & 11 Créditos internacionales para-CV. & Concepto: Social \\
\hline 11 Eólico. & 12 Créditos por fondos específicos para CV. & $\begin{array}{l}9 \text { En la sostenibilidad hay beneficios percibidos } \\
\text { por afiliados. }\end{array}$ \\
\hline $\begin{array}{l}12 \text { Equipos de alta eficiencia o } \\
\text { ecoeficientes. }\end{array}$ & $\begin{array}{l}13 \text { Fondos concursables no reembolsables para } \\
\mathrm{CV} \text {. }\end{array}$ & $\begin{array}{l}10 \text { Los asociados se han visto beneficiados por } \\
\text { el CV. }\end{array}$ \\
\hline 13 Fotovoltaico. & 14 Fondos de Infocoop para CV. & $\begin{array}{l}11 \text { Los procesos de CV han tenido impacto para } \\
\text { ser resilientes ante el covid-19. }\end{array}$ \\
\hline 14 Uso de biomasa. & 15 Fondos SBD para CV. & $\begin{array}{l}12 \text { Los procesos de CV han generado resiliencia } \\
\text { en tiempos de crisis. }\end{array}$ \\
\hline Concepto: Sellos o certificaciones verdes & 16 Ingresos por pagos vía PSA Fonafifo. & 13 Mejor calidad de vida de los asociados. \\
\hline $\begin{array}{l}15.15 \text { Carbono neutral y certificaciones } \\
\text { verdes. }\end{array}$ & 17 Otro tipo de crédito para impulsar CV. & 14 Nuevos empleos. \\
\hline 16 Certificaciones por eficiencia energética. & 18 Otro tipo de fondos para mejoras ambientales. & 15 Salarios sostenibles. \\
\hline
\end{tabular}

Se usó la siguiente fórmula para el análisis:

- Índice de impacto porcentual (\%) por variable $=($ total de puntos obtenidos $/ 30$ puntos $) * 100$.

Si para una variable A se obtuvo un puntaje de 30 y para variable B 20 puntos, se obtendrían los siguientes índices de gestión:

- Variable A: índice de impacto $100 \%$.

- Variable B: índice de impacto $66,7 \%$. 
Con este proceso de cálculo se obtuvieron tres tipos de índices de impacto, uno por cada eje temático: el índice de impacto en acciones verdes (IIACV), el índice de impacto en HF (IIHF) y el índice de impacto vinculado a los beneficios sostenibles (IIS).

\section{RESULTADOS}

Las organizaciones que fueron analizadas para valorar su accionar al inducir un CV tienen diversas características que las posicionan en sus sectores, en la tabla 3 se presentan variables que ayudan a comprender las dimensiones de su operación. La menos longeva es ASOELAGAR fundada en 1986, y las demás superan los 50 años de existencia, lo que las posiciona como entidades vinculadas a la economía de Costa Rica.

En la tabla 3 se presenta su actividad primaria, para unas es financiera y otras se centran en la producción y generación eléctrica. Las cooperativas de este estudio brindan servicios financieros como banca de segundo piso con fondos del Sistema de Banca para el Desarrollo (SBD), dado que este no es su fin, se ve como un beneficio a los afiliados. Se hace referencia en la tabla 3 que en el caso de la JUNTA UCR y ASOELAGAR sus servicios son exclusivos para los colaboradores de las organizaciones, por ello se les considera cerradas. Pero, las cooperativas sí brindan o intercambian bienes y servicios con sus afiliados y público externo.

Tabla 3. Datos y características de las organizaciones vinculadas a la ES

\begin{tabular}{|c|c|c|c|c|c|c|c|}
\hline Organización & $\begin{array}{l}\text { Año de } \\
\text { fundación }\end{array}$ & $\begin{array}{l}\text { Número de } \\
\text { afiliados }\end{array}$ & $\begin{array}{l}\text { Empleos } \\
\text { directos }\end{array}$ & $\begin{array}{l}\text { Tipo de } \\
\text { operación* }\end{array}$ & Patrimonio & $\begin{array}{l}\text { Acciones verdes } \\
\text { relevantes }\end{array}$ & Mercado** \\
\hline ASOELAGAR & 1986 & 700 & 3 & $\begin{array}{l}\text { Servicios } \\
\text { financieros. }\end{array}$ & ND & $\begin{array}{l}\text { Financió paneles de } \\
\text { celdas fotovoltaicas al } \\
\text { Grupo El Lagar. }\end{array}$ & $\begin{array}{l}\text { Cerrado a } \\
\text { los afiliados }\end{array}$ \\
\hline JUNTA UCR & 1940 & $\begin{array}{l}9000 \text { a } \\
10000 \\
\text { funcionarios } \\
\text { de la UCR }\end{array}$ & 74 & $\begin{array}{l}\text { Servicios } \\
\text { financieros. }\end{array}$ & ND & $\begin{array}{l}\text { Servicios financieros } \\
\text { verdes especializados } \\
\text { en construcción, autos y } \\
\text { equipos. }\end{array}$ & $\begin{array}{l}\text { Cerrado a } \\
\text { los afiliados }\end{array}$ \\
\hline Coopesantos & 1965 & 38.183 & 199 & $\begin{array}{l}\text { Producción de } \\
\text { energía eléctrica } \\
\text { por sistema } \\
\text { eólico. }\end{array}$ & $\begin{array}{l}34.267 \text { mil } \\
\text { millones de } \\
\text { colones en el año } \\
2020\end{array}$ & $\begin{array}{l}\text { Bonos de carbono } \\
\text { internacionales y } \\
\text { protección de recurso } \\
\text { hídrico }\end{array}$ & Nacional \\
\hline Coopedota & 1960 & 900 & 110 & $\begin{array}{l}\text { Producción de } \\
\text { café. }\end{array}$ & ND & $\begin{array}{l}\text { carbono neutral, } \\
\text { Alliance Rain Forest. }\end{array}$ & $\begin{array}{l}\text { Nacional e } \\
\text { internacional }\end{array}$ \\
\hline CoopeTarrazú & 1960 & 4650 & 312 & $\begin{array}{l}\text { Producción de } \\
\text { café. }\end{array}$ & $\begin{array}{l}6,763 \text { mil } \\
\text { millones de } \\
\text { colones al año } \\
2018\end{array}$ & $\begin{array}{l}\text { Alliance Rain Forest y } \\
\text { comercio justo, abono } \\
\text { orgánico, investigación y } \\
\text { desarrollo. }\end{array}$ & $\begin{array}{l}\text { Nacional e } \\
\text { internacional }\end{array}$ \\
\hline CoopeVictoria & 1943 & 2842 & 329 & $\begin{array}{l}\text { Producción de } \\
\text { café y azúcar. }\end{array}$ & $\begin{array}{l}14.133 \text { mil } \\
\text { millones de } \\
\text { colones al año } \\
2018\end{array}$ & $\begin{array}{l}\text { Carbono neutral, } \\
\text { "Alliance Rain Forest", } \\
\text { biocombustibles, } \\
\text { abono orgánico. }\end{array}$ & $\begin{array}{l}\text { Nacional e } \\
\text { internacional }\end{array}$ \\
\hline
\end{tabular}

ND: Los datos no están disponibles para el público en general, solo para afiliados.

* Se destacan las más relevantes para cada organización.

**Mercado se define como lugar donde colocar o vender productos o servicios, en el caso del solidarismo y la JUNTA UCR se enfocan en sus afiliados, por ello se denota como un mercado cerrado. 
En sus acciones verdes concretas, la selección de ASOELAGAR se dio al generar un modelo financiero particular, donde la asociación solidarista financió la compra de paneles de celdas fotovoltaicas para el Grupo El Lagar. De este modo, logró un beneficio mutuo en ambas entidades, ingresos financieros para la agrupación solidarista y ahorro en la factura eléctrica en el Grupo El Lagar. Este modelo puede ser replicado por empresas de capital privado al recibir recursos financieros de la asociación solidarista, en la cual los colaboradores recibirán beneficios directos de la operación y ayudarán a la organización privada a ser más competitiva. También, puede ayudar a las organizaciones privadas a buscar procesos de carbono neutralidad.

En el caso de la JUNTA UCR, se analizó su amplia cartera de servicios financieros verdes y sociales para sus afiliados, funcionarios de la UCR, los cuales incluyen casas ecoamigables, carros eléctricos o compras de equipos. También, esta organización brindó charlas en temas y ferias verdes, aunque por motivos de la pandemia ha enfocado su vinculación más hacia el ámbito de la salud.

Las cooperativas analizadas, al ser abiertas al mercado en términos de productos o servicios que ofrecen, se mostraron más dinámicas en cuanto a las certificaciones verdes que acompañan a sus productos y servicios en su mercadeo, además de buscar certificaciones en comercio justo o bonos de carbono que les retribuyan de forma directa con ingresos financieros para la sostenibilidad de su operación. Esto sin incluir otras acciones verdes que no generan recursos financieros directos, como la protección de mantos acuíferos y apoyo a la gestión de acueductos rurales.

Un total de 49 variables fueron analizadas por cada organización, de las cuales 16 pertenecieron al eje de acciones verdes, 18 al eje de HF y 15 al eje de sostenibilidad. En la figura 1 se presentan los resultados obtenidos para el eje de acciones concretas en CV y su impacto dentro de la organización. En las organizaciones de la ES no vinculadas al cooperativismo el impacto se concentró en pocas acciones, como en el tema de energías alternativas, manejo de residuos e infraestructura ecoeficiente para ASOELAGAR; mientras que la JUNTA UCR obtuvo resultados con el uso de equipos ecoeficientes y producción más limpia por poco uso de papel por la pandemia, el teletrabajo, metas internas para la reducción de desechos y campañas para reducir el consumo de agua. En estas organizaciones, dos variables con nulo impacto fueron los calentadores solares y la producción verde; la primera, al ser financiera se pensó que podía ser requerida por sus afiliados, y la segunda, explica el resultado por ser organizaciones de servicios financieros.

En las cooperativas las valoraciones fueron diversas en el impacto mediano y alto. En la figura 1 se pueden destacar las variables: búsqueda de certificaciones verdes o energéticas, protección de recurso hídrico, manejo de residuos, protección de la biodiversidad y apoyo a los afiliados en temas verdes (la educación en este punto es una actividad consistente). Se observaron en las cooperativas valoraciones de bajo impacto en las siguientes variables:

1) Calentadores solares. Estos productos no se contemplan dentro de esquemas operativos, aunque son los de mayor demanda en otros segmentos.

2) Empaques ecológicos. Su bajo impacto se visualizó cuando se respondió que, para ciertos productos como el café, aún no se dispone de un empaque ecoamigable que sustituya los actuales. También, la pandemia ha reducido envíos físicos de paquetería o similares.

3) Energía eólica. Este es un caso específico, solo Coopesantos R.L. la utiliza.

4) Construcción de infraestructura ecoeficiente. El rubro no mostró impacto en las organizaciones, pues no se detectaron proyectos de construcción ecoeficiente. 
Figura 1. Valoraciones de los impactos descritos por los representantes de las organizaciones de la ES en el eje de acciones para el CV, en la escala Likert.

แo Certricaciones en eficiencia energérica
in Carbono neutral en algün ärea

Uso de biomoso

Futovoltaico

$\frac{\pi}{6} \quad$ Equipus de alta eficienciau ecueficierites

Eólico

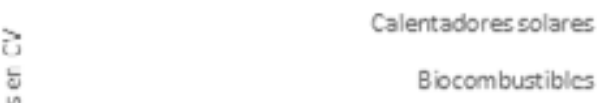

Producción verde o sustenible Producción más limpia Para protección de la biodnersidad $\frac{8}{8}$ Mancjo dd recur 30 hidrico Manejo de residuos

Empaqueseculúgicus Construcción de intraestructura ecoeficiente Apoyo a socios 0 afliados en temasverdes
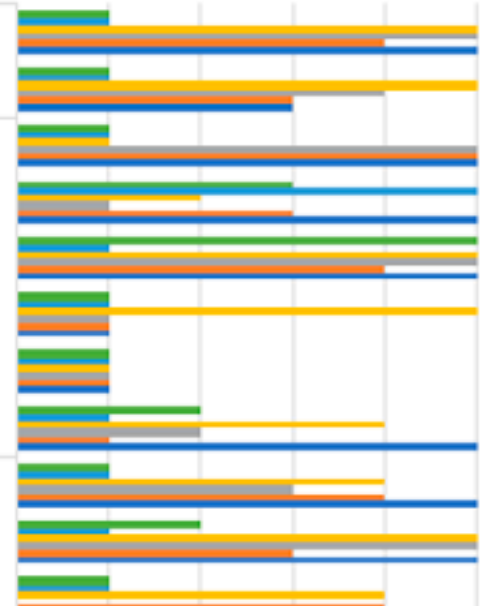

m JUNTA UCR

- asoelagar

n Coopesantos

n Coupeduta

" CoopeTarrazú

- Coopevictor a
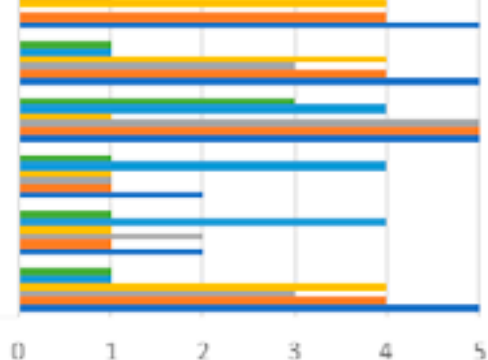

(1)

1 
En la figura 2 se describe el impacto que tuvieron las HF en las organizaciones. Para el caso de las organizaciones no vinculadas al cooperativismo, se observa que no logran tener acceso a HF, y sus acciones son financiadas por el capital propio de la organización. En las cooperativas el uso de capital propio es de alto impacto, algunas utilizan bonos de carbono, créditos especiales para acciones verdes, ingresos por premios derivados de certificaciones como comercio justo o apoyo directo del SBD. De forma general, el uso de HFV es limitado en todas las organizaciones. Dos variables consultadas, otro tipo de créditos para impulsar el CV y otro tipo de fondos para mejoras ambientales, no tuvieron valoraciones y se dejaron como no aplicables, por ello no fueron incluidas en las figuras.

Figura 2. Valoraciones de los impactos descritos por los representantes de las organizaciones de la ES en el eje temático HF, en la escala Likert.

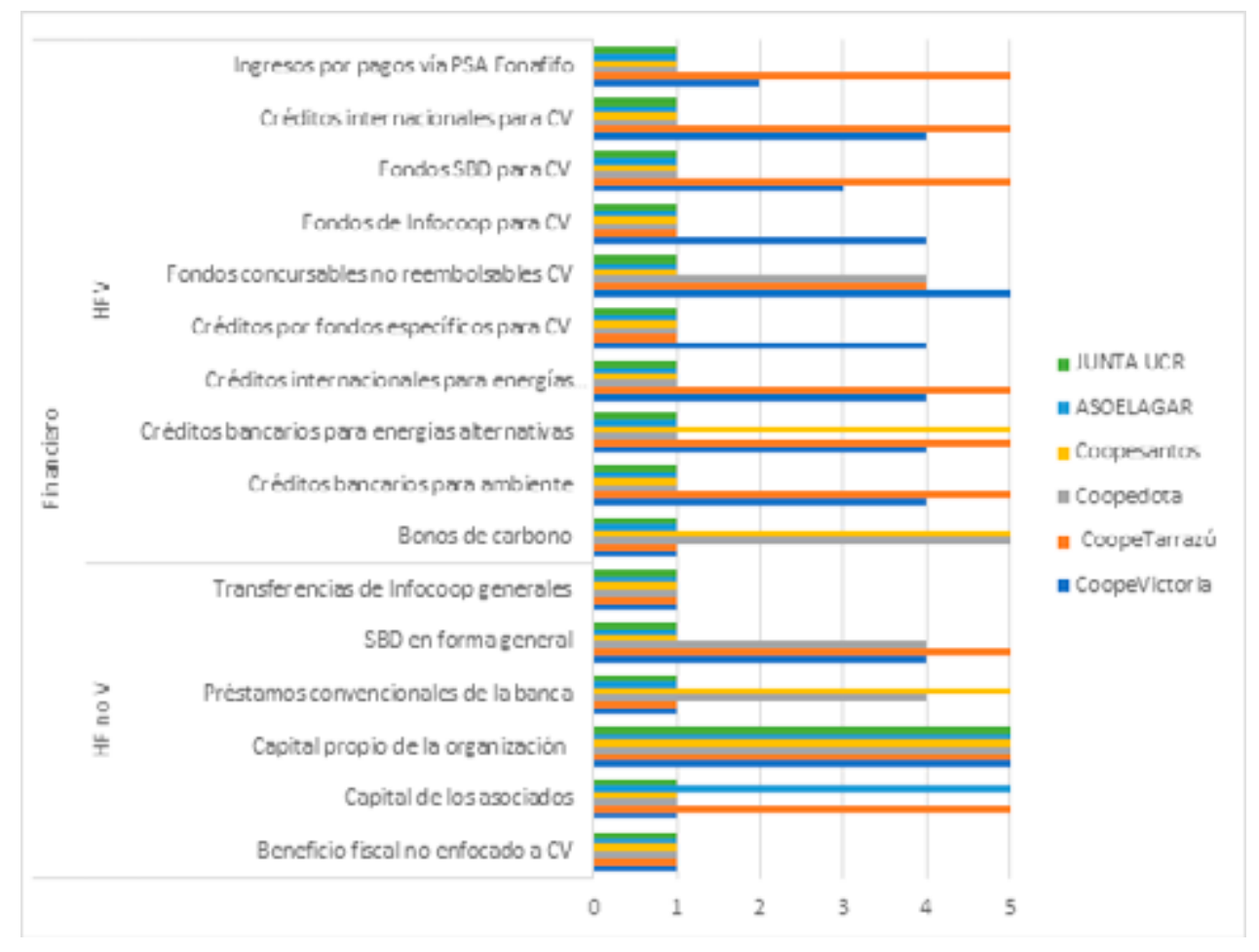


En la figura 3 se detalla cómo impactaron las variables vinculadas con la sostenibilidad en las organizaciones. Nuevamente, ASOELAGAR y JUNTA UCR son las que mostraron menor diversidad de impactos frente a las cooperativas, pero sí hay beneficios concretos en los conceptos económicos, sociales y ambientales para todas las organizaciones vinculadas con este estudio. Los afiliados a las cooperativas son los que perciben impactos medianos $\mathrm{y}$ altos en todas las variables analizadas.

Para el eje temático de acciones en CV se generó el IIACV. En la figura 4 se presenta el valor obtenido por cada variable, puede observarse que solo cuatro de las variables tuvieron un valor inferior al $50 \%$ (las vinculadas a infraestructura ecoeficiente, calentadores solares, energía eólica y empaques ecológicos). Se observan, con puntuaciones entre $50 \%$ y menos de $60 \%$, tres categorías que deberían tener más presencia: la protección a la biodiversidad, carbono neutralidad y biocombustibles. Todas estas acciones son de gran interés para las estrategias de descarbonización y economía circular, lo cual hace considerar que existe margen de mejora.

\section{Figura 3. Valoración del impacto en las organizaciones sobre el eje sostenibilidad, agrupadas en los} conceptos económico, social y ambiental, en la escala Likert.

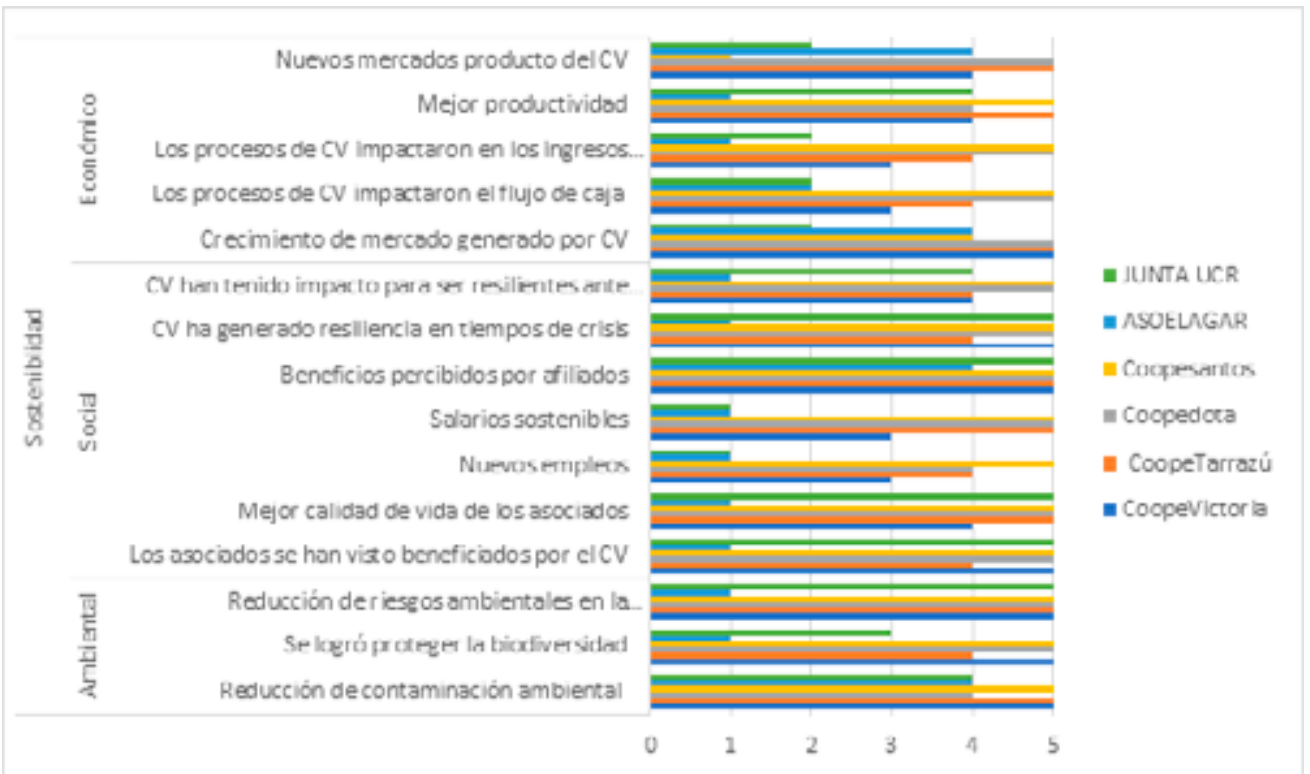

Utilizar el IIACV permite cuantificar las acciones y encontrar o detectar procesos que deberían ser reforzados para lograr un CV óptimo. El tema de la carbono neutralidad siempre es sensible, dado el costo económico del proceso de certificación. Este puede ser un elemento a considerar en las acciones directas con el SBD de Costa Rica, y conseguir certificaciones acompañadas de procesos de mejora en ecoeficiencia y reducción de emisiones en gases de efecto invernadero. Las certificaciones no pueden gestionarse sin HFV que estimulen las inversiones a tasas de interés éticas, pues los beneficios sociales de estas son amplios y se proyectan a futuro al descarbonizar las economías. 
Figura 4. Análisis de valoración porcentual del IIACV de las organizaciones.

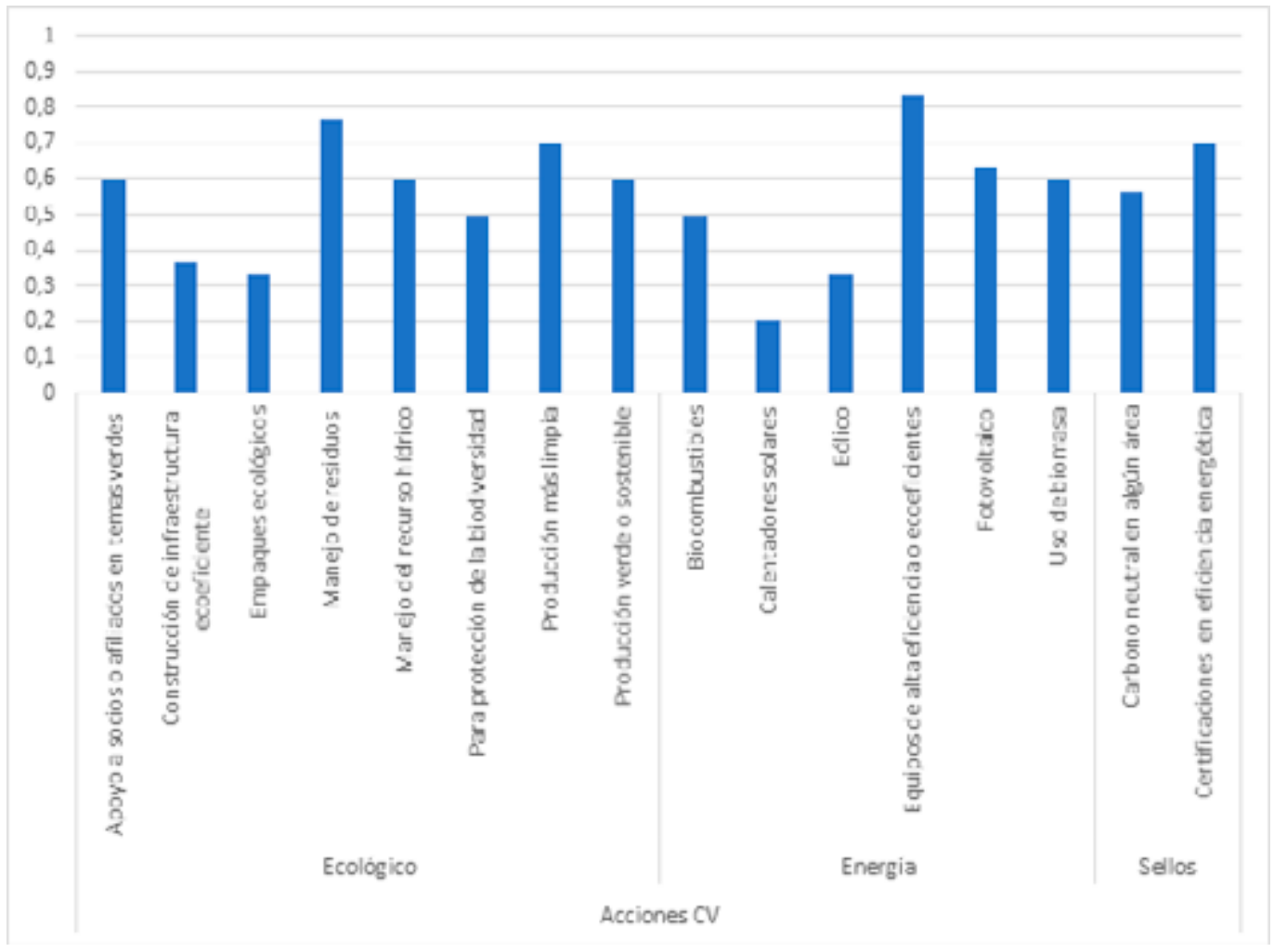


En la figura 5 se detallan los resultados de todas las variables del eje vinculado al impacto de las HFV y las HF convencionales o no verdes y su IIHF. Se observa que pocas obtuvieron valoraciones altas como el uso de capital propio $(100 \%)$. Solo otras tres variables apenas sobrepasaron el $50 \%$ del índice, por ejemplo, créditos específicos para energías alternativas y créditos del SBD. El uso de HFV y HF convencionales parece no generar un impacto en los casos analizados. Usar el IIHF expone con mayor claridad que se podría potenciar el CV si las organizaciones lograran acceder a HFV. En este indicador el margen para mejorar y crecer es elevado.

En el IIS en todas las variables se obtuvieron valores iguales o superiores el $60 \%$. Estos resultados se reflejan en la figura 6 . Es posible observar que la variable de sostenibilidad social vinculada a los beneficios que recibieron los afiliados supera el $90 \%$, la reducción de la contaminación ambiental fue de un $90 \%$ y los aportes para crecimiento en mercado de más de un $80 \%$, de este modo, los puntajes del desarrollo sostenible con respecto a lo económico, social y ambiental están en alta valoración. Debe notarse que las variables vinculadas con la resiliencia ante las crisis y el covid-19 mostraron valoraciones superiores al $75 \%$. Un punto donde las organizaciones pueden buscar una mejora es en la variable más baja relacionada con nuevos empleos, la cual obtuvo un $60 \%$.

Figura 5. Análisis de valoración porcentual del índice de impacto de las HFV y las HF no verdes de las organizaciones.

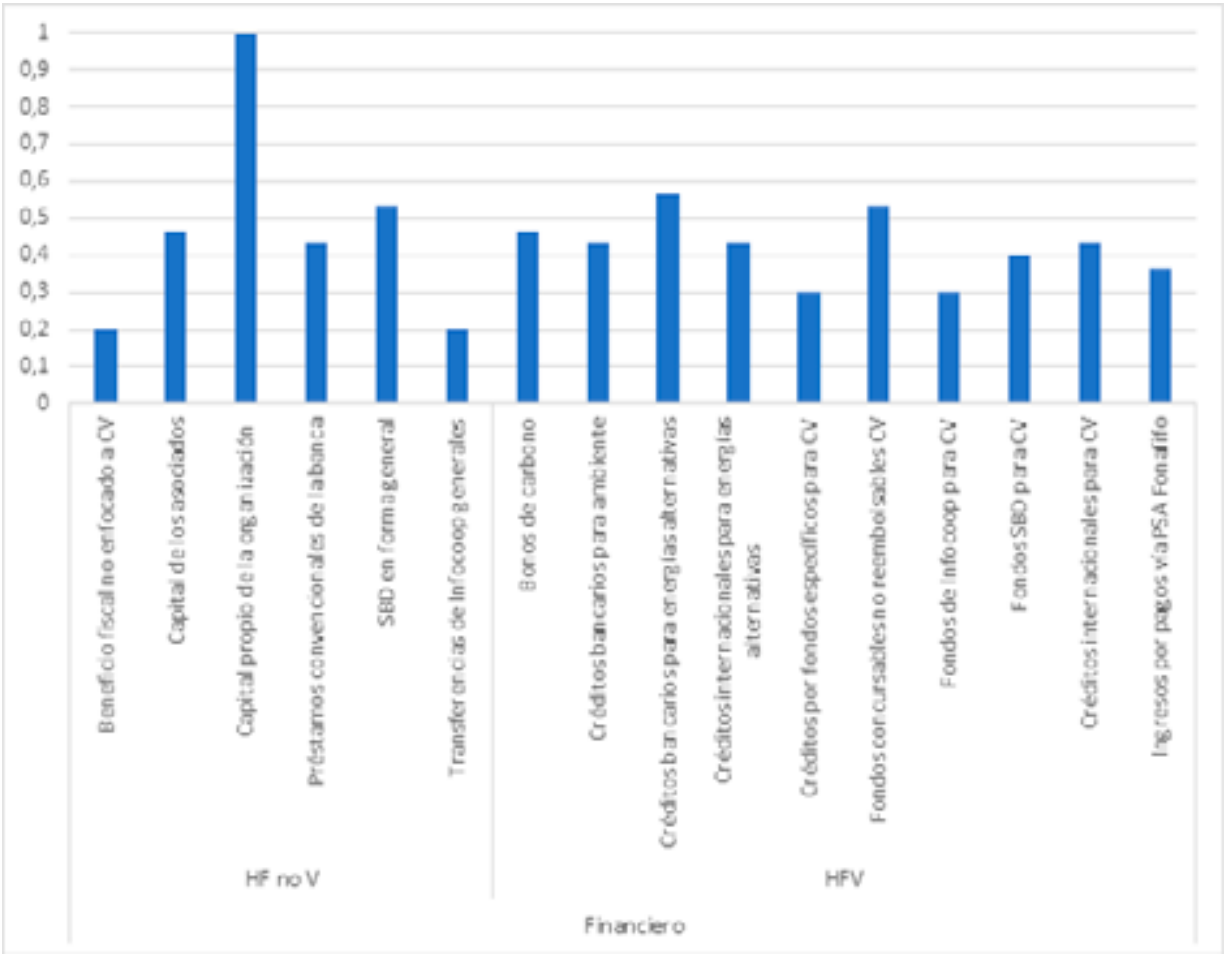


Figura 6. Análisis de valoración porcentual del IIS de las organizaciones.

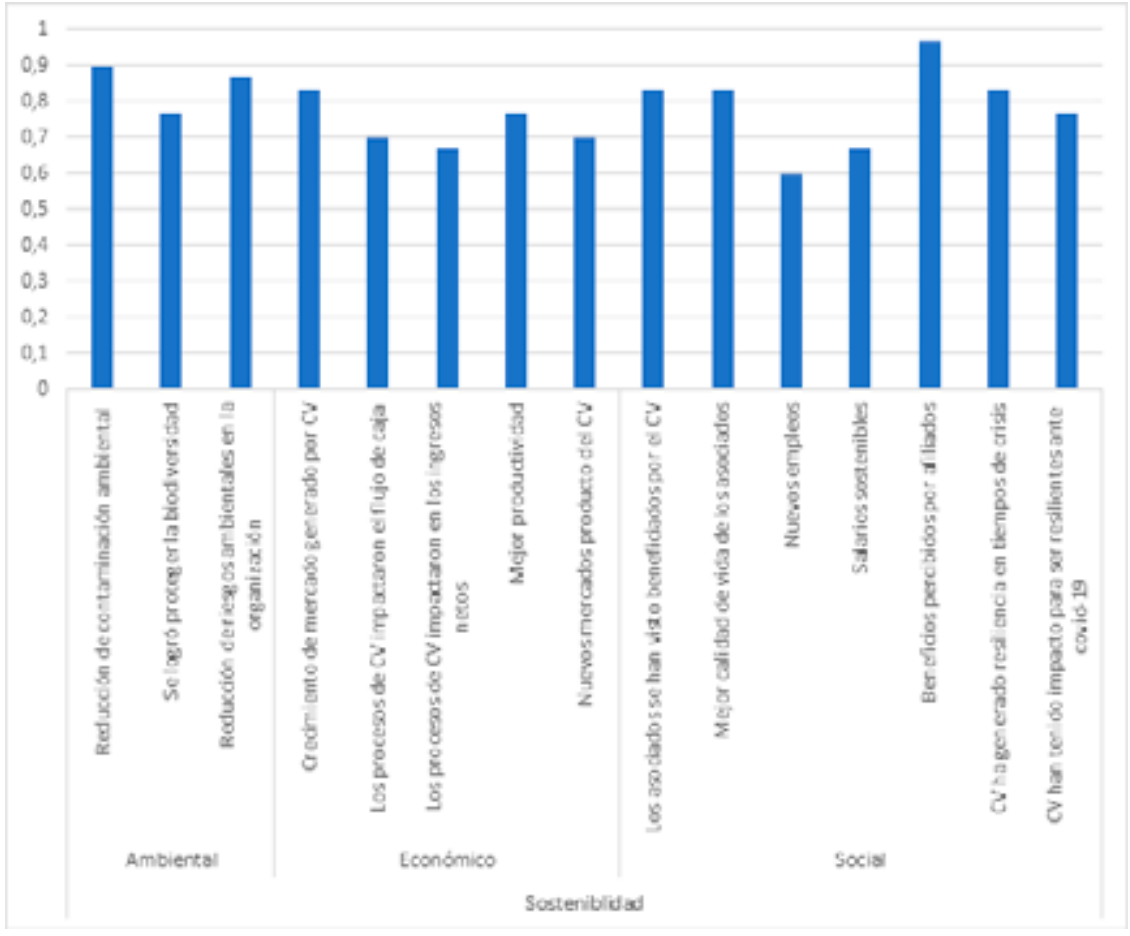

\section{CONCLUSIONES}

Las organizaciones vinculadas con la ES, en este estudio de casos, mostraron diferentes acciones y estrategias concretas para potenciar el CV. La poca diversidad de acciones para lograr un CV, dentro del solidarismo y las cajas de ahorro estudiadas, puede tener su justificación al ser organizaciones con funciones más cerradas, ya que tienen como población meta a los colaboradores o funcionarios de la empresa o universidad a la cual se vinculan. En el sector cooperativo, al tener la libertad de recibir afiliados y estar vinculado a la producción agrícola o de energía eléctrica (para los casos de estudio), sus acciones fueron más diversas, entre ellas la protección a la biodiversidad, protección de mantos acuíferos, conservación de bosques, uso de biomasa, por citar algunos ejemplos. El IIACV analizado muestra resultados en forma global y se observa un accionar fuerte en manejo de residuos, producción limpia y variables vinculadas con energía. Estas valoraciones son esperables, pues generan impactos financieros directos en las organizaciones que aplican esas medidas.

El empleo de HFV fue limitado en los casos en que el uso de capital propio parece ser dominante, aunque el acceso a este tipo de financiamiento está disponible en el país. Se puede considerar que para el caso de la JUNTA UCR y ASOELAGAR no les es posible aplicar fondos especiales verdes, y para las cooperativas en general parece que el uso de HFV no es potenciado. Este hallazgo merece un estudio a profundidad para conocer los motivos y razones del débil impacto y valoraciones obtenidas en el IIHF, salvo el caso de la variable de capital propio.

El eje vinculado con los beneficios sociales, económicos y ambientales de los procesos realizados para lograr un CV tiene un impacto superior al $60 \%$ en todas las variables analizadas, y en muchas superior al $80 \%$ al estudiar el IIS. También, se observa que estos procesos les hace ver como organizaciones con mayor resiliencia ante crisis y contra la generada por el covid-19. La información obtenida sirve de base para pensar en un estudio mayor, de modo que se pueda conocer si estos beneficios se asocian con el acceso a nuevos mercados verdes, expansión de productos, 
los beneficios derivados de las certificaciones verdes y los procesos de liderazgo que debieron desarrollar. Se deja también la inquietud de si al obtener HFV estos resultados podrían potenciarse a un nivel mayor.

Se concluye que las organizaciones analizadas en esta investigación de casos múltiples de la ES están vinculadas con el CV, y la conducción de estas se refleja en los resultados sobre los beneficios obtenidos. De manera diversa, han buscado migrar hacia la sostenibilidad económica, social y ambiental, pero se observa que hay vacíos y espacios por mejorar para lograr mayores impactos. Por último, la vinculación con HFV es muy limitada y deben explorarse las causas de estos resultados.

\section{Agradecimientos:}

Este trabajo se vincula con el trabajo doctoral el autor, se le agradece al comité de tesis, Dr. Marino Marozzi, Dra. Dalia Borge, Dra. Ivonne Scott y Dr. Fernando Zúñiga, del doctorado en Administración de Empresas de la UNED 


\section{REFERENCIAS}

Alianza Cooperativa Internacional [ACI]. (2021, 30 de enero). Identidad cooperativa: nuestros principios y valores. https:// www.ica.coop/es/cooperativas/identidad-alianza-cooperativa-internacional

Asociación Solidarista de Funcionarios de la Universidad Nacional [ASOUNA]. (2021). Asociación Solidarista de Funcionarios de la Universidad Nacional. https://www.asouna.una.ac.cr/es/conozcanos/documentos-de-interes

Baltodano, J. (2008). Los peligros del doble discurso, de lo internacional a lo local: el caso de la propuesta "Costa Rica Carbono Neutral 2021”. Economía y Sociedad, 33-34, 7-19.

Ben-Ner, A. (2018). Reflections on the future evolution of social, nonprofit and cooperative enterprise. Annals of Public and Cooperative Economics, 89, 109-124.

Bolsa Nacional de Valores [BNV]. (2021, 09 de julio). BNV sostenibilidad. https://www.bolsacr.com/empresas/ sostenibilidad-O

Bolton, P., Despres, M., Pereria da Silva, L., Samana, F. y Svartzman, R. (2020, enero). The Green Swan. Bank for International Settlements: www.bis.org

Campos-Rudin, M. (2019). Acoplamiento del producto interno bruto a la emisión de gases efecto invernadero: un limitante para lograr un crecimiento verde en Costa Rica. Revista Nacional de Administracion, 10, 77-92.

Clapp, C. (2018). Investing in a green future. Nature Climate Change, 8, 96-100.

Confederación Nacional de Asosiaciones Solidaristas [CONASOL]. (2021, 20 de marzo). Confederación Nacional de Asociaciones Solidaristas. https://conasol.cr/quienes-somos/que-es-solidarismo/

Contreras, G. (2012). Reseña histórica, Junta Administradora del Fondo de Ahorro y Préstamo de la Universidad de Costa Rica. San José: Imprenta Lil, S.A. http://www.jafapucr.com/Portals/1/Documentos

CoopeVictoria. (2019, diciembre). Acontecer Victoria, Boletín Oficial de CoopeVictoria R.L. http://www.coopevictoria. com/Publicaciones/

Dobles, R. (2021). El compromiso de Costa Rica: el camino a la neutralidad frente al carbono. https://www.un.org/es/ chronicle/article/el-compromiso-de-costa-rica-en-el-camino-hacia-la-neutralidad-frente-al-carbono

Fondo Nacional de Financiamiento Forestal [Fonafifo]. (2021, 8 de julio). Fondo Nacional de Financiamiento Forestal. Ministerio de Ambiente y Energía. http://www.fonafifo.go.cr

García-Barquero, R., Anda, J., Capote, C., Parra, T., Sanz, A. y Cuenca, M. (2018). Oportunidades de la bioeconomía circular en Andalucía. Bioeconomía y Desarrollo Sostenible. Mediterráneo Económico, 31, 119-131.

Instituto Nacional de Vivienda y Urbanismo [INVU]. (2021). Insituto Nacional de Vivienda y Urbanismo. https://www. invu.go.cr/

Junta Administrativa del Fondo de Ahorro y Préstamo de la Universidad de Costa Rica [JUNTA UCR]. (2021). Junta Administradora del Fondo de Ahorro y Préstamo de la Universidad de Costa Rica. https://www.jafapucr.com/

Marcuella, C. y Saz, M. (2008). Los principios cooperativos facilitadores de la innovación: un modelo teórico. Revista de Estudios Cooperativos (Revesco) (94), 59-79.

Martínez, A. (2015). Las cooperativas y su acción sobre la sociedad. Revesco, 117, 34-49.

Martínez-Salgado, C. (2012). El muestreo en investigación cualitativa: principios básicos y algunas controversias. Cienc. Saúde Coletiva, 17, 613-619.

Ministerio de Ciencia, Innovación Tecnología y Telecomunicaciones [Micitt]. (2020). Estrategia nacional de bioeconomía, Costa Rica 2020-2030. ttps://www.micit.go.cr/sites/default/files/estrategia_nacional_bioeconomia_cr_ corregido.pdf

Ministerio de Ambiente y Energía [Minae]. (2018). Plan de Descarbonización. https://minae.go.cr/images/pdf/Plande-Descarbonizacion-1.pdf

Montolio, J. (2002). Economía social: concepto, contenido y significación en España. CIRIEC-España, Revista de Economía Pública, Social y Cooperativa, 42, 5-31. 
Naciones Unidas. (2015, 25 de septiembre). Objetivos para el desarrollo sostenible. http://www.un.org/ sustainabledevelopment/es/objetivos-de-desarrollo-sostenible/

Organización para la Cooperación y el Desarrollo Económicos [OCDE]. (2011, 11 de mayo). Toward green growth economy. ://dx.doi.org/10.1787/9789264111318-en

OCDE. (2017). Green Growth Indicators. http://dx.doi.org/10.1787/9789264268586-en

Peters, G., Andrew, R., Canadell, J., Friedlingstein, P., Jackson, R., Korsbankken, J. y Peregon, A. (2019, 4 de diciembre). Carbon dioxide emissions continue to grow and slowly emerging climate policies. Nature Climate Change. doi:10.1038/s41558-019-0659-6

Programa de las Naciones Unidas para el Desarrollo [PNUD]. (2018). Manual de Biofin. Iniciativa de finanzas para la biodiversidad. New York: Programa de Naciones Unidas para el Desarrollo. http://www.biodiversityfinance.org

Promotora del Comercio Exterior de Costa Rica [Procomer]. (2021, 17 de marzo). Programa de crecimiento verde. https://www.procomer.com/noticia/comprador-internacional-noticia/programa-de-crecimiento-verdecontinua-apoyando-empresas-con-fondos-no-reembolsables/

Quesada, A. (2008). El “tercer sector”, la "Economía Social” y el trabajo social en Costa Rica. Revista de Ciencias Sociales, 119, 107-119.

Ruiz, L. y Retolaza, J. (2007). Análisis comparativo de la banca ética con la banca tradicional: identificación de indicadores. En Conocimiento, innovación y emprendedores: camino al futuro (págs. 1104-1118). España: Universidad de la Rioja, Grupo de investigación FEDRA. https://dialnet.unirioja.es/servlet/articulo?codigo=223333 1

Salinas, F. (2003). Economía Social una forma diferente de hacer empresa. Sociedad y Utopía, Revista de Ciencias Sociales(22), 97-116.

Sánchez, O. y Navarrete, G. (2017). La experiencia de Costa Rica en el pago de servicios ambientales: 20 años de lecciones aprendidas. Revista de Ciencias Ambientales, 51, 195-214.

Sasia, P. (2008). Entender hoy la banca ética. Revista Internacional de Estudios Vascos, 53, 507-532.

Soto, W. (1987). Para entener al movimiento solidarista costarricense. Anuario de Estudios Centroamericanos, Universidad de Costa Rica, 13, 113-125.

Stern, N. (2010, 7 de abril). El informe Stern sobre la Economía del Cambio Climático. https://webarchive.nationalarchives. gov.uk/+/http://www.hm-treasury.gov.uk/sternreview_index.htm

The Board of the Millenium Ecosystem Assesment. (2005, 01 de marzo). Millenium ecosystem assesment. http://www. wri.org/publication/millennium-ecosystem-assessment-living-beyond-our-means 